\title{
Toward the Development of Preventive Interventions to Reduce HRSB, HIV/AIDS, and Multiple Problem Behaviors
}

\author{
Gilbert J. Botvin • Kenneth W. Griffin
}

Published online: 25 January 2014

(C) Society for Prevention Research 2014

While we have witnessed tremendous progress in the field of prevention science over the years, some important gaps remain. This Supplemental Issue helps advance the field by focusing on preventive interventions for children and adolescents, and the potential for long-term effects on drug abuse and health-risking sexual behaviors (HRSB) in the late adolescent and young adult years. It not only expands our understanding of the etiology and prevention of HRSB and HIV/ AIDS but also has important implications for the development of interventions capable of widespread dissemination and an impact on multiple health risk behaviors.

The six papers included in the Supplemental Issue represent a broad range of intervention strategies and methods, supported by diverse theoretical frameworks, and focus on target populations that vary in terms of demographic characteristics and level of baseline risk. One paper focuses on a school-based intervention targeting early aggressive behavior in children (Kellam et al. 2014). Two papers focus on universal interventions targeting drug use and associated risk factors using either a combined school- and family-based intervention implemented during childhood (Hill et al. 2014) or a familybased intervention implemented during early adolescence (Spoth et al. 2014). One paper focuses on universal and targeted interventions implemented during childhood to address conduct problems using both school- and family-based approaches (Conduct Problems Prevention Research Group 2014). Two others involve selective family-based interventions implemented either alone during childhood (Skinner et al. 2014) or as part of a multilevel strategy combining universal, selective, and indicated approaches implemented

G. J. Botvin $(\bowtie) \cdot K$. W. Griffin

Division of Prevention and Health Behavior, Department of Public

Health, Weill Cornell Medical College, 402 East 67th Street, New

York, NY 10065, USA

e-mail: gjbotvin@med.cornell.edu during early adolescence (Caruthers et al. 2014). The interventions were tested using different study designs, research methods, and data analysis approaches.

Despite these notable differences and without any direct focus on sexual behavior, four of these long-term studies found significant (direct or indirect) reductions in HRSB for some or all of participants (Caruthers et al. 2014; Hill et al. 2014; Kellam et al. 2014; Spoth et al. 2014). Although the remaining two studies did not find intervention effects, they did provide etiologic data supporting the general thesis of this Supplemental Issue (Conduct Problems Prevention Research Group 2014; Skinner et al. 2014). As a group, this collection of papers makes an important contribution to the prevention literature by providing long-term intervention and/or etiologic evidence that early preventive interventions not explicitly targeting sexual behavior or HRSB can prevent risk behaviors associated with the acquisition of HIV/AIDS. Moreover, these papers provide additional evidence that preventive interventions targeting shared risk and protective factors can have an impact on multiple problem behaviors.

The findings from this impressive collection of studies are consistent with a large body of research focusing on (1) epidemiology findings regarding the developmental course of risk behaviors, (2) research on common psychosocial risk and protective factors at multiple levels of influence that contribute to problem behaviors, and (3) research demonstrating that intervening on shared risk and protective factors can prevent multiple risk behavior outcomes.

\section{Developmental Course of Risk Behaviors}

Individual studies and literature reviews have shown that multiple risk behaviors and outcomes are often observed in the same individual children or adolescents (Biglan et al. 2004; Elliott et al. 1989; Greenberg and Lippold 2013). For 
children, these may include antisocial behavior, conduct problems, academic difficulties, delinquency, aggression, bullying, and mental health problems. For adolescents, these may also include alcohol, tobacco, other drug use, early sexual activity, and risky sexual behaviors. Youth who engage in multiple risk behaviors account for a large proportion of these behaviors as well as to higher health-care expenditures, lost productivity, reduced quality of life, and criminal justice system involvement (Biglan et al. 2004).

Many health risk behaviors occur during adolescence and can be best understood within a developmental framework. As children transition into adolescence, they begin to make independent decisions about their own behaviors, including those related to substance use, sexual activity, and other types of risky behaviors. During this time, young people strive for independence and self-definition and are increasingly influenced by peers. They may be susceptible to a variety of negative societal messages, media portrayals, adult role models, and peers that have a significant impact on decisions about their own behavior. Furthermore, adolescents typically face several academic, social, and vocational challenges and explore new roles during the adolescence years. Some youth will handle developmental challenges more successfully than others, and negative outcomes may be more likely among youth who are less successful in conventional pursuits. Youth who struggle with developmental tasks are more vulnerable to negative social influences and are more likely to engage in risk behaviors.

\section{Common Risk and Protective Factors}

A common set of etiological factors has been found to be associated with multiple problem behaviors, including risk and protective factors at multiple levels of influence (i.e., individual, family, peer, school, and community), as well as genetic and neurobiological factors. While no single factor or pathway serves as a necessary and sufficient condition leading to multiple problem behaviors, these behaviors are the result of a multivariate mix of factors. Individual-level risk factors include depression, anxiety, poor coping skills, negative life events, susceptibility to peer pressure, desire for social acceptance, low self-esteem, and poor impulse control. Family-level risk factors include poor parent-child bonding or communication, inadequate parental monitoring, and family conflict. At the peer-group level, risk factors include association with deviant peers and/or rejection by conforming peers as well as modeling of problem behaviors. At the school and community levels, some key risk factors include lack of bonding to school or neighborhood, disorganized community, and economic adversity. Protective effects also occur at these same levels of influence, including the individual level (e.g., good executive functioning skills), at the family level (e.g., positive attachments and close relationships with parents and siblings, involvement in prosocial, and conventional activities), and at the community level (e.g., a supportive/nurturing environment and opportunities for educational and economic advancement).

A common thread through the literature regarding the prevention of problem behavior among youth is the need to enhance levels of psychosocial resilience by promoting protective factors and reducing susceptibility to social risk factors. At the individual level, resilience may involve teaching a general set of social and self-management skills. At the family level, this may involve teaching family communication skills and parenting skills. Interventions that promote resilience offer the potential of preventing a variety of problem or health risk behaviors that share a similar constellation of risk and protective factors. To the extent that preventive interventions increase competent skills and success in developmental tasks, they also may promote other positive outcomes such as academic achievement.

\section{Targeting Shared Risk and Protective Factors}

Prevention approaches are often narrowly focused on a limited set of proximal etiologic factors specific to the targeted risk behavior or problem (e.g., tobacco, illicit drug use, HIV/AIDS, and violence), with separate approaches designed to target each risk behavior or problem. Early prevention initiatives typically had a limited focus on cognitive and/or attitudinal factors. For example, early smoking prevention programs were designed to increase knowledge of the adverse consequences of cigarette smoking and promote anti-smoking attitudes. Advances in etiology research and the concomitant development of prevention theory now indicate that many problem behaviors share similar etiologic factors and developmental trajectories. This not only suggests the possibility that some prevention approaches may have a positive effect on empirically and theoretically related risk behaviors not explicitly targeted by the intervention but also suggests the potential of developing preventive interventions that - by targeting a set of shared etiologic factors - can prevent multiple problem behaviors. Such an approach would be more efficient, costeffective, and more likely to be adopted and implemented. This would facilitate the adoption and sustained use of effective prevention approaches and ultimately lead to reduced mortality and morbidity.

Our own work with the Life Skills Training program provides an example of a broad-based intervention that can prevent multiple problem behaviors (see Botvin and Griffin 2010 for a review). Originally designed to prevent cigarette smoking, Life Skills Training targets an array of risk and 
protective factors associated with several different problem behaviors. In addition to effects on cigarette smoking, studies show it can prevent alcohol use, binge drinking, the use of marijuana and other illicit drugs, risky driving, delinquency, violence, and HRSB.

\section{Implications}

The findings from this Supplemental Issue demonstrate the potential of preventive interventions delivered in childhood or early adolescence for producing long-term effects on HRSB and other risk behaviors for HIV/AIDS. These effects are particularly noteworthy because none of the interventions had a direct focus on sexual behavior. This has both practical and scientific implications. On a practical level, since program content related to sex or HRSB can often be a source of sensitivity (particularly for interventions targeting children) and may serve as a barrier to implementation in some communities, interventions such as the ones discussed in this Supplemental Issue have a greater likelihood of wide dissemination. On a scientific level, these findings expand the knowledge base relating to the prevention of HRSB and HIV/AIDS. Moreover, these findings provide additional support for the thesis that broad-based preventive interventions targeting shared risk and protective factors can have an impact on multiple problem behaviors. Rather than developing and implementing separate programs (all competing for time and resources) that target proximal risk factors, carefully constructed broad-based prevention approaches may be able to reduce multiple risk behaviors in a way that is efficient, cost-effective, and produce a greater public health impact. More research is needed to examine the convergence of risk and protective factors across domains and the potential of interventions for preventing multiple health risk behaviors. Finally, this Supplemental Issue demonstrates the value of carefully designed, methodologically rigorous longitudinal prevention research and can serve both as a source of inspiration and as a foundation for future advances in prevention science.
Financial Disclosure Dr. Botvin is the President of the National Health Promotion Associates (NHPA), which markets the Life Skills Training program. Dr. Griffin is a consultant to NHPA.

\section{References}

Biglan, A., Brennan, P. A., Foster, S. L., \& Holder, H. D. (2004). Helping adolescents at risk: Prevention of multiple problem behaviors. New York: Guilford.

Botvin, G. J., \& Griffin, K. W. (2010). Advances in the science and practice of prevention: Targeting individual-level etiologic factors and the challenge of going to scale. In L. M. Scheier (Ed.), Handbook of drug use etiology: Theory, methods, and empirical findings (pp. 631-650). Washington, DC: American Psychological Association.

Caruthers, A. S., Van Ryzin, M. J., \& Dishion, T. J. (2014). Preventing high-risk sexual behavior in early adulthood with family interventions in adolescence: Outcomes and developmental processes. Prevention Science, 15. doi:10.1007/s11121-013-0383-9.

Conduct Problems Prevention Research Group. (2014). Trajectories of risk for early sexual activity and early substance use in the Fast Track prevention program. Prevention Science, 15. doi:10.1007/ s11121-012-0328-8.

Elliott, D. S., Huizinga, D., \& Menard, S. (1989). Multiple problem youth: Delinquency, substance use, and mental health problems. New York: Springer.

Greenberg, M. T., \& Lippold, M. A. (2013). Promoting healthy outcomes among youth with multiple risks: Innovative approaches. Annual Review of Public Health, 34, 253-270.

Hill, K. G., Bailey, J. A., Hawkins, J. D., Catalano, R. F., Kosterman, R., Oesterle, S., \& Abbott, R. D. (2014). The onset of STI diagnosis through age 30: results from the Seattle Social Development Project intervention. Prevention Science, 15. doi:10.1007/s11121-013-0382-x.

Kellam, S. G., Wang, W., Mackenzie, A. C. L., Brown, C. H., Ompad, D. C., Or, F., Ialongo, N. S., Poduska, J. M., \& Windham, A. (2014). The impact of the Good Behavior Game, a universal classroom based preventive intervention in first and second grades, on high-risk sexual behaviors and drug abuse and dependence disorders into young adulthood. Prevention Science, 15. doi:10.1007/s11121-012-0296-z.

Skinner, M. L., Fleming, C. B., Haggerty, K. P., \& Catalano, R. F. (2014). Sex risk behavior among adolescent and young adult children of opiate addicts: Outcomes from the Focus on Families prevention trial and an examination of childhood and concurrent predictors of sex risk behavior. Prevention Science, 15. doi:10.1007/s11121-012-0327-9.

Spoth, R., Clair, S., \& Trudeau, L. (2014). Universal family-focused intervention with young adolescents: Effects on health-risking sexual behaviors and STDs among young adults. Prevention Science, 15. doi:10.1007/s11121-012-0321-2. 